\title{
HÁBITOS ALIMENTARIOS DEL BAGRE “LIFE” Trichomycterus punctulatus (VALENCIENNES, 1846) (ACTINOPTERYGII, SILURIFORMES) EN EL RÍO PISCO, PERÚ
}

\author{
FEEDING HABITS OF THE CATFISH Trichomycterus punctulatus \\ (VALENCIENNES, 1846) (ACTINOPTERYGII, SILURIFORMES) FROM PISCO \\ RIVER, PERÚ
}

\author{
Adrián Vera Arabe ${ }^{1}$, Eduardo Oyague Passuni ${ }^{2}$, Liz Castañeda Córdova ${ }^{3}$ y Zulema Quinteros Carlos $^{4}$
}

\begin{abstract}
Resumen
Trichomycterus punctulatus es un bagre de agua dulce de hábitos bentopelágicos, endémico de los ríos de la vertiente occidental del territorio peruano. Se trata de un depredador situado en la cima de la cadena trófica y como tal, podría constituir un buen integrador de la información ecológica, por lo que generar información sobre esta especie contribuiría con un mejor entendimiento del ensamblaje comunitario, además de propiciar su conservación y manejo. Los hábitos alimentarios de $T$. punctulatus se determinaron analizando el contenido estomacal de 127 individuos colectados durante dos estaciones del año: seca (Agosto, 2010) y húmeda (Mayo, 2011), en 3 sectores (6 estaciones de muestreo) de la cuenca media del río Pisco. Los ítems alimenticios, fueron identificados a nivel de familia. En base a estos datos se calcularon el Índice de Importancia Relativa (IRI) y el Índice de Importancia Numérica (INI), encontrándose que $T$. punctulatus consume principalmente individuos de las familias Chironomidae, Hydropsychidae y Leptohyphidae durante la temporada seca; mientras que en la temporada húmeda, consume individuos de las familias Chironomidae y Elmidae. Mediante el índice lineal de selección de alimentos $(L)$, se determinó que $T$. punctulatus no presenta una selección activa, siendo más bien oportunista. Con el desarrollo del método gráfico de Cortés (1997), se determinó que la estrategia alimentaria de $T$. punctulatus es generalista. Por último con un análisis de Kruskal-Wallis se determinó que existe variación en los hábitos alimentarios por sector de cuenca y, entre las temporadas seca y húmeda.
\end{abstract}

Palabras clave: Trichomycterus punctulatus, dieta, selectividad, estrategia alimentaria, río Pisco.

\begin{abstract}
Trichomycterus punctulatus is an endemic benthopelagic freshwater catfish found in the western systems of Peru; it is a predator and as such is a good integrator of ecological information. The information generated from this species will improve a better understanding of community structure and promote its conservation and management. Feeding habits of T. punctulatus were determined by analyzing the stomach contents of 127 individuals collected in two seasons: dry (August, 2010) and wet (May, 2011), in three sectors (6 sampling stations) of the Pisco river middle basin. Feeding items were set at the family level, and the Index of Relative Importance (IRI) and the Index of Numerical Importance (INI) were calculated. It was found out that $T$. punctulatus mainly consumes individuals from the Chironomidae, Hydropsychidae and Leptohyphidae families during the dry season; whereas in the wet season, it consumes individuals from the Chironomidae and Elmidae families. Using the linear food selection index $(L)$, it was determined that $T$. punctulatus is not an active selector, being rather opportunistic. The graphical method of Cortés (1997), determined that the feeding strategy of T. punctulatus is a generalist. Finally with a Kruskal-Wallis analysis, it was determined that there are changes in feeding habits within basin sectors and among dry and wet seasons.
\end{abstract}

Key words Trichomycterus punctulatus, diet, selectivity, feeding strategy, Pisco river.

Introducción.

En los sistemas acuáticos, los peces ocupan un importante lugar en la cadena trófica, pues consumen una gran variedad de presas y muestran gran cantidad de hábitos alimentarios; dependiendo su supervivencia, crecimiento y reproducción de la energía y los nutrientes de su alimento (Lampert \& Sommer, 2007). 
Trichomycterus punctulatus (Valenciennes, 1846) es un bagre de agua dulce bentopelágico endémico, que se encuentra presente en la mayoría de las cuencas de la vertiente occidental peruana (Burgess, 1989; Reis et al., 2003; Ferraris, 2007), y a pesar de ser una especie de consumo en la región (Zacarías, 2008), poco se sabe acerca de su biología en general.

A través de esta investigación se busca generar un nuevo conocimiento sobre los hábitos alimentarios de T. punctulatus (Valenciennes), incrementando así la información disponible para la especie y produciendo datos que puedan ser útiles para investigaciones posteriores en lo que respecta a sus patrones de uso del hábitat, consumo de recursos $\mathrm{y}$ viabilidad poblacional.

En la actualidad se desconoce si Trichomycterus punctulatus (Valenciennes) presenta selectividad en sus hábitos alimentarios, o si muestra alguna estrategia alimentaria en particular. Así mismo, se plantea la hipótesis de que sus hábitos alimentarios varían a lo largo del espacio y tiempo en la cuenca del río Pisco, el cual podría estar siendo afectado por actividades antrópicas como a regulación hídrica, la agricultura, la ganadería, el comercio y el transporte.

\section{Materiales y métodos.}

Se trabajó con un total de 127 individuos (Figura 1) colectados en el río Pisco en seis estaciones (Figura 2, Tabla 1) (Vera et al., 2012), a cada uno de los cuales se le extrajo el estómago siguiendo las recomendaciones de Murphy \& Willis (1996). Posteriormente, el contenido estomacal (macroinvertebrados bentónicos básicamente) fue separado y preservado con alcohol de $70^{\circ}$ en viales Eppendorf. La identificación del contenido estomacal se realizó hasta el nivel taxonómico de Familia u Orden utilizando un estereomicroscopio OLYMPUSSZ51, y basándose en claves especializadas en macroinvertebrados acuáticos de la región neotropical, como: Roldán (1988), Heckman (2001; 2002; 2003; 2006; 2008), Domínguez et al.(2006) y Domínguez \& Fernández (2009).

Tabla 1. Número de estómagos analizados por temporada y estación. Río Pisco. Perú

\begin{tabular}{lcc}
\hline & $\begin{array}{c}\text { Temporada Húmeda } \\
\text { (Mayo, 2011) }\end{array}$ & $\begin{array}{c}\text { Temporada Seca } \\
\text { (Agosto, 2010) }\end{array}$ \\
\cline { 2 - 3 } Estación Alta & 26 & 29 \\
Estación Media & 15 & 32 \\
Estación Baja & 10 & 15 \\
\hline
\end{tabular}

Con los datos obtenidos de la identificación y cuantificación del contenido estomacal de $T$. punctulatus (Valenciennes), se calcularon los valores de porcentaje de ocurrencia $(O \%$, número de estómagos que contienen la categoría alimenticia expresado en porcentaje del total de estómagos), porcentaje por número $(N \%$, número de individuos de cada categoría alimenticia expresado en porcentaje del total de individuos de todas las categorías alimenticias) y porcentaje de composición por peso ( $W \%$, peso de cada categoría alimenticia expresado en porcentaje del peso total de todas las categorías alimenticias) (Hyslop, 1980). Para el cálculo de $W \%$, primero se calculó una longitud promedio del largo del cuerpo (L) para cada ítem alimenticio, dato que fue utilizado en la estimación del peso mediante el uso de la relación alométrica: $\mathrm{W}=\mathrm{aL}^{\mathrm{B}}$ usando las constantes a y b provistas por Benke et al. (1999) y Miserendino (2001) para las familias identificadas de macroinvertebrados bentónicos.

Una vez obtenidos los datos de $O \%, N \%$ y $W \%$, se procedió a hallar el Índice de Importancia Relativa (IRI) $I R I=(\% N+\% W) x \% O($ Pinkas et al., 1971) y el Índice de Importancia Numérica (INI)

$$
I I N=\sqrt{\% \text { composición numérica } x \% \text { ocurrencia }}
$$

(Windell, 1968) como medida estándar para comparar las categorías alimenticias.

Los datos del muestreo de macroinvertebrados bentónicos fueron obtenidos siguiendo las recomendaciones de Oyague (2009). Con esta información y con los datos de identificación de los estómagos, se procedió a calcular el índice de selección lineal de alimento $(L) L=r_{i}-p_{i}$ (Strauss, 1979) por temporada.

Para determinar la estrategia alimenticia se desarrolló el modelo gráfico de Cortés (1997), usando los datos de $O \%, N \%$ y $W \%$.

Por último, para comprobar el grado de diferencia dietaria, se realizó un análisis Cluster y un análisis de varianza no paramétrico de Kruskal Wallis (Glantz, 2005) con el programa PAST (Hammer et al., 2001), para lo que se realizó un pre-tratamiento de datos que consistió en eliminar a unos pocos individuos con valores susceptibles a incrementar la heterogeneidad de la data. De este modo, de ocurrir diferencia significativa entre los grupos, se debería enteramente a los datos en su conjunto y no al efecto de valores atípicos.

\section{Resultados y discusión.}

\section{Caracterización de la dieta}

La dieta de $T$. punctulatus (Valenciennes) estuvo constituida en total por 13 ítems alimenticios, 12 ítems correspondientes a macroinvertebrados bentónicos: Baetidae, Leptohyphidae, Simuliidae, Chironomidae, Diptera indeterminados ${ }^{1}$, Corydalidae, Elmidae, Coleoptera indeterminados ${ }^{2}$ Hydropsychidae, Hydroptilidae, Hydrobiosidae, Trichoptera indeterminados $^{3}$ y un vertebrado: Rhinella limensis

\footnotetext{
${ }^{1}$ Diptera distintos de Chironomidae

${ }^{2}$ Coleoptera distintos de Elmidae

${ }^{3}$ Trichoptera distintos de Hydropsychidae, Hydroptilidae e Hydrobiosidae
} 
Tabla 2. Índice de Importancia Relativa (IRI) en temporada seca (Agosto, 2010) y temporada húmeda (Mayo 2011). Río Pisco. Perú.

\begin{tabular}{lcccccccccc}
\hline & \multicolumn{2}{c}{ O\% } & \multicolumn{2}{c}{ N\% } & \multicolumn{2}{c}{ W\% } & \multicolumn{2}{c}{ IRI } & \multicolumn{2}{c}{ IRI\% } \\
& Temp. & Temp. & Temp. & Temp. & Temp. & Temp. & Temp. & Temp. & Temp. & Temp. \\
& Seca & Húmeda & Seca & Húmeda & Seca & Húmeda & Seca & Húmeda & Seca & Húmeda \\
\hline Baetidae & 59.211 & 5.882 & 7.243 & 0.680 & 6.441 & 0.251 & 810.253 & 5.481 & 6.820 & 0.124 \\
Leptohyphidae & 84.211 & 19.608 & 12.336 & 4.989 & 6.631 & 1.115 & 1597.276 & 119.672 & 13.445 & 2.704 \\
Simuliidae & 80.263 & 17.647 & 9.818 & 2.948 & 1.376 & 0.172 & 898.448 & 55.052 & 7.562 & 1.244 \\
Chironomidae & 88.158 & 41.176 & 50.957 & 56.689 & 2.477 & 1.146 & 4710.678 & 2381.436 & 39.651 & 53.807 \\
Corydalidae & 14.474 & 7.843 & 0.246 & 0.907 & 61.109 & 93.543 & 888.032 & 740.784 & 7.475 & 16.738 \\
Elmidae & 35.526 & 37.255 & 0.952 & 26.757 & 0.039 & 0.459 & 35.200 & 1013.947 & 0.296 & 22.909 \\
Hydropsychidae & 88.158 & 9.804 & 15.896 & 2.041 & 15.447 & 0.824 & 2763.111 & 28.089 & 23.258 & 0.635 \\
Hydroptilidae & 19.737 & 3.922 & 0.884 & 0.907 & 0.053 & 0.023 & 18.505 & 3.646 & 0.156 & 0.082 \\
Hydrobiosidae & 25.000 & 1.961 & 0.694 & 0.227 & 4.122 & 0.560 & 120.412 & 1.542 & 1.014 & 0.035 \\
Coleoptera indet. & 2.632 & 1.961 & 0.034 & 0.454 & 0.002 & 0.012 & 0.094 & 0.913 & 0.001 & 0.021 \\
Diptera indet. & 21.053 & 15.686 & 0.448 & 3.175 & 0.528 & 1.557 & 20.548 & 74.215 & 0.173 & 1.677 \\
Trichoptera indet. & 7.895 & 1.961 & 0.493 & 0.227 & 1.773 & 0.339 & 17.885 & 1.110 & 0.151 & 0.025 \\
\hline
\end{tabular}

(Werner). Este último ítem constituye un registro atípico, puesto que se encontraron 3 individuos de esta especie en un único estómago, que fue excluido para los análisis posteriores a fin de no generar conclusiones sesgadas debido a su rareza y a la gran influencia que producía en los cálculos relacionados con biomasa.

La Tabla 2 muestra los valores del Índice de Importancia Relativa (IRI, en porcentaje) para la temporada seca, donde se puede apreciar que los ítems que dominan la dieta con más del $75 \%$ son: Chironomidae (39.65\%), Hydropsychidae (23.26\%) y Leptohyphidae (13.45\%), todos con un rango de importancia relativa secundaria (Olaya-Nieto et al., 2009). Mientras que Baetidae (6.82 \%), Simuliidae (7.56\%) y Corydalidae (7.48\%) suman el 21.86\%; dejando a los ítems restantes (Elmidae, Hydroptilidae, Hydrobiosidae, Coleoptera indet., Diptera indet. y Trichoptera indet.) el $1.79 \%$. Mientras que durante la temporada húmeda es posible observar que los ítems dominantes son: Chironomidae (53.81\%), Elmidae $(22.91 \%)$ y Corydalidae $(16.74 \%)$. Estos tres ítems suman más del $90 \%$ del porcentaje de IRI (IRI\%), dejando tan solo el $6.55 \%$ a los ítems restantes (Baetidae, Leptohyphidae, Simuliidae, Hydropsychidae, Hydroptilidae, Hydrobiosidae, Coleoptera indet., Diptera indet. y Trichoptera indet.) como se puede apreciar en la Figura 3.

Los resultados del cálculo del Índice de Importancia Numérica (INI) se detallan en la Tabla 3 para las temporadas seca y húmeda. Los tres ítems más importantes para la temporada seca fueron Chironomidae, Hydropsychidae y Leptohyphidae con valores de 67.02, 37.43 y 32.23 unidades, respectivamente; por otro lado, para la temporada húmeda los dos ítems más importantes fueron Chironomidae con un valor de 48.31 unidades y Elmidae, con 31.57 unidades. En la Figura 4 se puede visualizar la variación en la preferencia alimenticia del T. punctulatus (Valenciennes) para ambas temporadas.

Además se puede apreciar que en ambas temporadas los quironómidos son el ítem más consumido por $T$. punctulatus (Valenciennes), y junto con Hydropsychidae y Leptohyphidae componen más del $75 \%$ de la dieta en la temporada seca y, junto con Elmidae y Corydalidae más del $90 \%$ en la temporada húmeda. Si bien Macdonald \& Green (1983) argumentaron que el uso de índices compuestos aporta poca información adicional en los estudios de dieta, su uso en la actualidad se fundamenta en eliminar las faltas de información que presenta el análisis de los métodos de ocurrencia, número y peso por sí solos (Cortés, 1997; Cortés, 1998; Habit et al., 2005; Manoni et al., 2009).

Los datos del INI muestran básicamente los mismos resultados que el IRI a pesar de que no toma en cuenta la composición por peso en su algoritmo, siendo esta importante por agregar valor sobre el contenido calorífico de la presa. A pesar de que ambos índices muestran resultados similares, su utilización por parte de los investigadores suele ser motivada por una decisión arbitraria, habitualmente en función al hecho de que el INI puede ser utilizado de manera más directa con los datos crudos, mientras que el IRI requiere un paso posterior a la simple identificación donde se pesa o calcula el volumen de las presas consumidas. Cortés $(1997 ; 1998)$ recomienda el uso del IRI pues toma en cuenta la variable peso o volumen del ítem consumido, variable que a su vez puede ser usada en análisis posteriores.

De acuerdo a los resultados de estos dos índices (excluyendo del análisis a Rhinella limensis (Werner) por motivos ya sustentados); la dieta de $T$. punctulatus (Valenciennes) se compone principalmente de individuos de las familias Chironomidae, Hydropsychidae y Leptohyphidae para la temporada seca, mientras que para la temporada húmedalas familias predominantes son: Chironomidae y Elmidae. 
Tabla 3. Índice de Importancia Numérica (INI) en T. seca (Agosto 2010) y T. húmeda (Mayo 2011). Río Pisco. Perú.

\begin{tabular}{lcccccc}
\hline & \multicolumn{2}{c}{$\% \mathrm{O}$} & \multicolumn{2}{c}{$\% \mathrm{~N}$} & \multicolumn{2}{c}{ INI } \\
& Temp. Seca & Temp. Húmeda & Temp. Seca & Temp. Húmeda & Temp. Seca & Temp. Húmeda \\
\hline Baetidae & 59.211 & 5.882 & 7.243 & 0.680 & 20.709 & 2.000 \\
Leptohyphidae & 84.211 & 19.608 & 12.336 & 4.989 & 32.231 & 9.890 \\
Simuliidae & 80.263 & 17.647 & 9.818 & 2.948 & 28.071 & 7.213 \\
Chironomidae & 88.158 & 41.176 & 50.957 & 56.689 & 67.024 & 48.314 \\
Corydalidae & 14.474 & 7.843 & 0.246 & 0.907 & 1.888 & 2.667 \\
Elmidae & 35.526 & 37.255 & 0.952 & 26.757 & 5.814 & 31.573 \\
Hydropsychidae & 88.158 & 9.804 & 15.896 & 2.041 & 37.435 & 4.473 \\
Hydroptilidae & 19.737 & 3.922 & 0.884 & 0.907 & 4.178 & 1.886 \\
Hydrobiosidae & 25.000 & 1.961 & 0.694 & 0.227 & 4.166 & 0.667 \\
Coleoptera indet. & 2.632 & 1.961 & 0.034 & 0.454 & 0.297 & 0.943 \\
Diptera indet. & 21.053 & 15.686 & 0.448 & 3.175 & 3.070 & 7.057 \\
Trichoptera indet. & 7.895 & 1.961 & 0.493 & 0.227 & 1.972 & 0.667 \\
\hline
\end{tabular}

Estos resultados son similares a los reportados por Manoni (2009) para T. corduvensis (Weyenbergh), donde los quironómidos también presentaron los mayores valores del índice, seguidos por Oligochaeta y Baetidae Así mismo, la dieta de T. punctulatus (Valenciennes) es similar a la reportada para la especie chilena T. aerolatus (Valenciennes), donde los ítems más consumidos fueron: Chironomidae, Baetidae y Elmidae (Habit et al., 2005). Del mismo modo, Scott et al. (2007) reportan una dieta similar para T. chiltoni (Eigenmann), compuesta principalmente por quironómidos y efemerópteros. Por otro lado, Román-Valencia (2001) reporta una dieta para T. caliense (Eigenmann) donde las larvas de coleópteros son las de mayor ocurrencia, seguida por individuos del orden Diptera y Trichoptera. Como se ha podido observar en la comparación con estudios similares enfocados en congéneres de $T$. punctulatus (Valenciennes), éstos son predominantemente insectívoros, por lo que la ocurrencia de 3 especímenes de Rhinella limensis (Werner) en un estómago podría estar brindando información sobre un comportamiento oportunista de T. punctulatus.

\section{Análisis de selectividad}

Siguiendo las recomendaciones de Habit et al. (2005) sólo se tomaron para el cálculo las presas con abundancias relativas mayores al $1 \%$ tanto en los ítems de la dieta como en los ofertados en el ambiente.

El índice de selección lineal por temporada seca (Tabla 4) muestra que T. punctulatus (Valenciennes) presenta selección para los ítems Chironomidae (0.319 unidades) y Baetidae (0.051 unidades), donde este último presenta un valor bastante bajo para la calificación de selección. Una selección neutra para Leptohyphidae, con un valor de $L$ igual a 0.011 unidades y no selección para Simuliidae e Hydropsychidae con valores de -0.127 y -0.130 unidades, respectivamente.

Para la temporada húmeda se observó una alta selectividad para Chironomidae ( $L=0.556$ unidades), selectividad neutra para Simuliidae $(L=0.007$ unidades) y no selectividad para Elmidae ( $L=-0.228$ unidades). Estos resultados pueden ser revisados en la Tabla 4.

Tabla 4. Índice de selección linear de Strauss. Río Pisco. Perú.

\begin{tabular}{lccc}
\hline & \multicolumn{3}{c}{ Temporada Seca (Agosto, 2010) } \\
\cline { 2 - 4 } İtem alimenticio & $\begin{array}{c}\mathrm{A} \% \\
\text { estomago } \\
\left(r_{\mathrm{i}}\right)\end{array}$ & $\begin{array}{c}\mathrm{A} \% \\
\text { hábitat } \\
\left(p_{\mathrm{i}}\right)\end{array}$ & $\begin{array}{c}\text { Selectividad } \\
\text { Strauss } \\
\left(L=r_{i}-p_{i}\right)\end{array}$ \\
\hline Baetidae & 0.073 & 0.022 & 0.051 \\
Leptohyphidae & 0.125 & 0.114 & 0.011 \\
Simuliidae & 0.099 & 0.226 & -0.127 \\
Chironomidae & 0.515 & 0.196 & 0.319 \\
Hydropsychidae & 0.161 & 0.290 & -0.130 \\
\hline & \multicolumn{3}{c}{ Temporada Húmeda (Mayo, 2011) } \\
\cline { 2 - 4 } & $\mathrm{A} \%$ & $\mathrm{~A} \%$ & Selectividad \\
Ítem alimenticio & estomago & hábitat & Strauss \\
& $\left(r_{\mathrm{i}}\right)$ & $\left(p_{\mathrm{i}}\right)$ & $\left(L=r_{i}-p_{i}\right)$ \\
\hline Simuliidae & 0.030 & 0.023 & 0.007 \\
Chironomidae & 0.589 & 0.023 & 0.566 \\
Elmidae & 0.278 & 0.506 & -0.227 \\
\hline
\end{tabular}

Si bien se puede decir si existe selectividad o no, se puede observar en la Tabla 3 que, a excepción de un par de casos, los valores obtenidos para el índice de selección lineal se encuentran cerca de los límites sugeridos por Habit et al. (2005), lo que significa que la selección o no selección es baja, inclinándose más hacia valores neutros de selección o selección aleatoria.

La no selección que podemos observar en Elmidae, posiblemente se deba a que estos son individuos completamente esclerotizados, difíciles de digerir, y al parecer poco palatables para $T$. punctulatus (Valenciennes). En cambio, en Chironomidae es posible observar un alto valor de selección, probablemente debido a que estos son individuos abundantes, pequeños y poco móviles, que además de presentar una masa considerable por su gran número, serían altamente palatables debido a su 
cuerpo blando y poco esclerotizado (solo una parte de la cabeza).

Dados los bajos valores con los que la mayoría de los ítems alimenticios alcanzó la categoría de selección o no selección, se puede decir que Trichomycterus punctulatus (Valenciennes), en general, no presenta una activa selección de los ítems consumidos y al parecer tiende a consumir los macroinvertebrados que están más disponibles en el ambiente. Estos resultados concuerdan con los observados por otros investigadores (Habit et al., 2005; Chará et al., 2006; Scott et al., 2007; Manoni et al., 2009). Aun así, esta generalización no parece aplicarse a Simuliidae en temporada seca, ni a Elmidae en temporada húmeda, donde en ambos casos, a pesar de su alta disponibilidad en el ambiente, son poco consumidos.

\section{Análisis de estrategia alimentaria}

Como se puede apreciar en la Figura 5, de acuerdo a Cortés (1997), para la temporada seca T. punctulatus (Valenciennes) presenta como ítems de consumo raros a Coleoptera indeterminados, Trichoptera indeterminados, Diptera indeterminados, Elmidae, Hydroptilidae e Hydrobiosidae. Además, estos se encuentran ubicados sobre el eje de porcentaje de composición por número, lo que indica que sus cantidades influyen más que su peso en la dieta. Por otro lado, se encuentran Baetidae, Simuliidae, Leptohyphidae e Hydropsychidae, donde la ubicación espacial de estos datos los define como de una dieta generalista, evidenciada en que la que la mayoría de los estómagos analizados, presentaron varios de estos ítems pero en baja abundancia. Chironomidae también es considerado como un ítem de consumo generalista, y aporta poca composición por peso pero es consumido en gran cantidad por la mayoría de los individuos de $T$. punctulatus (Valenciennes). Por último, Corydalidae representa un ítem de dieta especializada, constituida por pocos individuos que elevan la composición en peso del contenido estomacal.

En la Figura 6, se puede observar que en la temporada húmeda los ítems alimenticios Coleoptera indeterminados, Trichoptera indeterminados, Diptera indeterminados, Hydrobiosidae, Hydroptilidae, Hydropsychidae, Baetidae, Simuliidae y Leptohyphidae, se encuentran como ítems de consumo raro, donde su composición por número es más importante (aun siendo baja) que su composición por peso. Elmidae y Chironomidae se encuentran en una región no definida por Cortés (1997), pero pueden ser considerados ítems de consumo generalista, siendo su composición por número y ocurrencia importante pero sin relevancia en composición por peso. Como era de esperarse en el caso de Corydalidae, se incluye en una dieta especializada, al igual que en la temporada seca.

Los resultados en ambas temporadas muestran que T. punctulatus (Valenciennes) tiene una dieta generalista, pues los ítems considerados raros indicarían que los consume si están disponibles en el medio. Esto concuerda con los resultados obtenidos para T. corduvensis (Weyenbergh) (Manoni et al., 2009) y T. areolatus (Valenciennes) (Habit et al., 2005), donde les describen una conducta oportunista. Para reforzar esto se puede citar la ocurrencia de tres individuos de Rhinella limensis (Werner) encontrados en uno de los estómagos analizados, que al parecer fueron consumidos por el simple hecho de que este individuo tenía la capacidad de hacerlo, dado que presentaba una longitud total más grande que la longitud máxima reportada en la bibliografía (Reis et al., 2003), y también debido a que este ítem estaba disponible, ya que no existe reporte de consumo del orden anura por algún pariente cercano de Trichomycterus punctulatus (Valenciennes). Así mismo, el consumo de Corydalidae por parte de pocos individuos de $T$. punctulatus (Valenciennes) indica que fueron accesibles o pudieron cazarlos.

Análisis de variación espacial y temporal de la dieta

La hipótesis nula a probar fue que no existe diferencia en la dieta entre la temporada seca y húmeda. Los valores de $p$ del análisis por sector de cuenca como por temporada pueden ser revisados en el Tabla 5.

Los resultados de la prueba estadística muestran que existen diferencias significativas en la dieta de $T$. punctulatus (Valenciennes); esto puede apreciarse gráficamente en la Figura 7 para las temporadas, y en mayor detalle (entre sectores de cuenta) en la Tabla 5. Luego del análisis de selectividad y estrategia alimenticia, era de esperarse este resultado pues $T$. punctulatus (Valenciennes) muestra una estrategia generalista, aprovechando los recursos disponibles en

Tabla 5. Valores de $p$ del análisis de Kruskal Wallis. Río Pisco. Perú.

\begin{tabular}{cccc}
\hline \multicolumn{3}{c}{ Por sector de cuenca } & Por temporada \\
Seca \\
Alta Seca & Media Seca & Baja Seca & \\
vs & vs & vs & vs \\
Alta Húmeda & Media Húmeda & Baja Húmeda & Húmeda \\
\hline 0.001996 & 0.002853 & 0.02796 & 0.0007868 \\
\hline
\end{tabular}

el momento y lugar que emplea para forrajear.

\section{Conclusiones.}

1. Según el Índice de Importancia Relativa (IRI), la dieta del bagre $T$. punctulatus (Valenciennes) se compone principalmente de quironómidos, e individuos de las familias Hydropsychidae y Leptohyphidae para la temporada seca; mientras que para la temporada húmeda consume individuos de las familias Chironomidae, Elmidae y Corydalidae. 
2. Según el Índice de Importancia Numérica (INI), la dieta de $T$. punctulatus (Valenciennes) se basa en individuos de las familias Chironomidae, Hydropsychidae y Leptohyphidae en la temporada seca; mientras que para la temporada húmeda, de individuos de las familias Chironomidae y Elmidae.

3. Tanto los análisis por temporada como por sector de cuenca muestran básicamente los mismos resultados; en general, Trichomycterus punctulatus (Valenciennes) es oportunista, no presenta una activa selección de los ítems consumidos y al parecer tiende a consumir los macroinvertebrados que están más disponibles en el ambiente.

4. Según el análisis gráfico de estrategia alimenticia, Trichomycterus punctulatus (Valenciennes) presenta una estrategia alimenticia de tipo generalista, básicamente consumiendo lo que encuentre en el ambiente, tanto en temporada seca como húmeda.

5. Empleando la prueba estadística de Kruskal Wallis, se puede afirmar, con un nivel de confianza del $95 \%$, que existen diferencias en lo espacio-temporal en la dieta de $T$. punctulatus (Valenciennes).

\section{Agradecimientos.}

A las empresas TgP y Knight Piésold por apoyar en todo momento a esta investigación. A los laboratorios de la División de Limnología y Entomología de CORBIDI; al Laboratorio de Ecología de Procesos (LEP) y al Museo de Entomología Klaus Raven Büller de la UNALM, por ofrecer tanto los instrumentos como el apoyo para la toma de datos de este proyecto. A Enith Sifuentes y Lucia Castillo por su ayuda en la versión en inglés del resumen. A Hiromi Yagui por su invaluable ayuda en el proceso de identificación.

\section{Literatura citada.}

Benke A.C., Huryn A.D., Smock L.A. \& Wallace J.B. 1999. Length-Mass Relationships for Freshwater Macroinvertebrates in North America with Particular Reference to the Southeastern United States. Journal of the North American Benthological Society. 18: 308-343.

Burgess W.E. 1989. An atlas of freshwater and marine Catfishes. TFH Publications. 784.

Cortés E. 1997. A critical review of methods of studying fish feeding based on analysis of stomach contents: application to elasmobranch fishes. Canadian Journal of Fisheries and Aquatic Sciences. 54: 726- 738. 1998. Methods of studying fish feeding: reply. Canadian Journal of Fisheries and Aquatic Sciences. 55: 2706-2707.

Chará J.D., Baird D.J., Telfer T.C. \& Rubio E.A. 2006. Feeding ecology and habitat preferences of the catfish genus Trichomycterus in low-order streams of the Colombian Andes. Journal of Fish Biology. 68: 10261040.
Domínguez E., Molineri C., Pescador M., Hubbard M. \& Nieto C. 2006. Ephemeroptera of South America. Pensoft Publishers. Sofia-Moscow. 646.

Domínguez E. \& Fernández H.R. 2009. Macroinvertebrados Bentónicos Sudamericanos. Sistemática y Biología. Fundación Miguel Lillo. San Miguel de Tucumán. 253.

Ferraris C.J. 2007. Checklist of catfishes, recent and fossil (Osteichthyes: Siluriformes), and catalogue of siluriform primary types. Zootaxa. 1418: 628

Glantz S.A. 2005. Primer of Biostatistics: Sixth Edition. McGraw-Hill Companies, Incorporated.

Habit E., Victoriano P. \& Campos H. 2005. Ecología trófica y aspectos reproductivos de Trichomycterus areolatus (Pisces,Trichomycteridae) en ambientes lóticos artificiales. Revista de Biología Tropical. 53: 195-210.

Hammer Ø., Harper D.a.T. \& Ryan P.D. 2001. PAST: Paleontological Statistics Software Package for Education and Data Analysis. Palaeontología Electrónica 4(1): 9 pp. http: //palaeoelectronica.org/ $2001 \mathrm{1} / \mathrm{past} /$ issue 1 01.htm.

Heckman C.W. 2001. Encyclopedia of South American Aquatic Insects: Collembola. Illustrated Keys to Known Families, Genera, and Species in South America. Springer. Dordrecht, The Netherlands. 418. . 2002. Encyclopedia of South American Aquatic Insects: Ephemeroptera. Illustrated Keys to Known Families, Genera, and Species in South America. Springer. Dordrecht, The Netherlands. 428. 2003. Encyclopedia of South American Aquatic Insects: Plecoptera. Illustrated Keys to Known Families, Genera, and Species in South America. Springer. Dordrecht, The Netherlands. 338.

2006. Encyclopedia of South American Aquatic Insects: Odonata - Anisoptera. Illustrated Keys to Known Families, Genera, and Species in South America. Springer. Dordrecht, The Netherlands. 725. . 2008. Encyclopedia of South American Aquatic Insects: Odonata - Zygoptera. Illustrated Keys to Known Families, Genera, and Species in South America. Springer. Dordrecht, The Netherlands. 687.

Hyslop E.J. 1980. Stomach contents analysis - a review of methods and their application. Journal of Fish Biology. 17: 411-429.

Lampert W. \& Sommer U. 2007. Limnoecology. The Ecology of Lakes and Streams. Oxford University Press. Oxford. 324.

Macdonald J.S. \& Green R.H. 1983. Redundancy of Variables Used to Describe Importance of Prey Species in Fish Diets. Canadian Journal of Fisheries and Aquatic Sciences. 40: 635-637.

Manoni R., Garelis P., Tripole E. \& Vallania E. 2009. Diet and feeding preferences of Trichomycterus corduvensis Weyenbergh, 1877 (Siluriformes, Trichomycteridae) in two rivers of the Quinto River basin (San Luis, Argentina). Biological Limnology. 21: 161-167.

Miserendino M.L. 2001. Length-Mass relationships for Macroinvertebrates in Freshwater Environments of Patagonia (Argentina). Ecología Austral. 11: 3-8.

Murphy B.R. \& Willis D.W. 1996. Fisheries techniques, $2^{\circ}$ edition. American fisheries society. Bethesda, Maryland. 732.

Olaya-Nieto C., Soto-Fernández P. \& Barrera-Chica J. 2009. Hábitos alimentarios de la Mayupa (Sternopygus 
macrurus Bloch \& Schneider, 1801) en el río Sinú, Colombia. Revista MVZ Córdoba. 14: 1787-1795.

Oyague Passuni, E. 2009. Discusión sobre el Número de Unidades Muestrales y Tamaño de Unidad Muestral para la estimación de Valores de Densidad de Macroinvertebrados Bentónicos en Ambientes Lóticos. Ecología Aplicada. 8: 61-70.

Pinkas L., Oliphant M.S. \& Iverson I.L.K. 1971. Food habits of Albacore, Bluefin Tuna, and Bonito in California Waters. California Fish Game. 152: 1-105.

Reis R.E., Kullander S.O. \& Ferraris C.J. 2003. Check List of the Freshwater Fishes of South and Central America. Pontifícia Universidade Católica do Rio Grande do Sul (EDIPUCRS). Porto Alegre. 742.

Roldán G. 1988. Guía para el estudio de los macroinvertebrados acuáticos del Departamento de Antióquia. Colciencia / Fen Colombia/ Universidad de Antioquia. Medellín. 217.

Román-Valencia C. 2001. Ecología trófica y reproductiva de Trichomycterus caliense y Astroblepus cyclopus (Pisces: Siluriformes) en el río Quindio, Alto Cauca, Colombia. Revista de Biología Tropical (International Journal of Tropical Biology). 49: 657-666.
Scott S., Pardo R. \& Vila I. 2007. Trophic niche overlap between two Chilean endemic species of Trichomycterus (Teleostei: Siluriformes). Revista Chilena de Historia Natural. 80: 431-437.

Strauss R.E. 1979. Reliability Estimates for Ivlev's Electivity Index, the Forage Ratio, and a Proposed Linear Index of Food Selection. Transactions of the American Fisheries Society. 108: 344-352.

Vera A. 2012. Hábitos alimenticios del bagre "life" Trichomycterus punctulatus (Actinopterygii, Siluriforme) en el río Pisco, Perú. Tesis para optar el Título de Biólogo. Universidad Nacional Agraria la Molina. 64.

Windell J.T. 1968. Food analysis and rate of digestion. Páginas: 197-203. En: Ricker WE (ed.). Methods for assessment of fish production in freshwater (IBP Handbook $\mathrm{N}^{\mathrm{o}}$ 3). Blackwell Scientific Publications. Oxford, Inglaterra.

Zacarías S. 2008. Determinación de los desembarques y esfuerzos pesqueros en los recursos continentales. Monitoreo Limnológico pesquero en la costa norte del Perú. IMARPE. Lima. 44.

\section{FIGURAS CITADAS EN EL TEXTO}

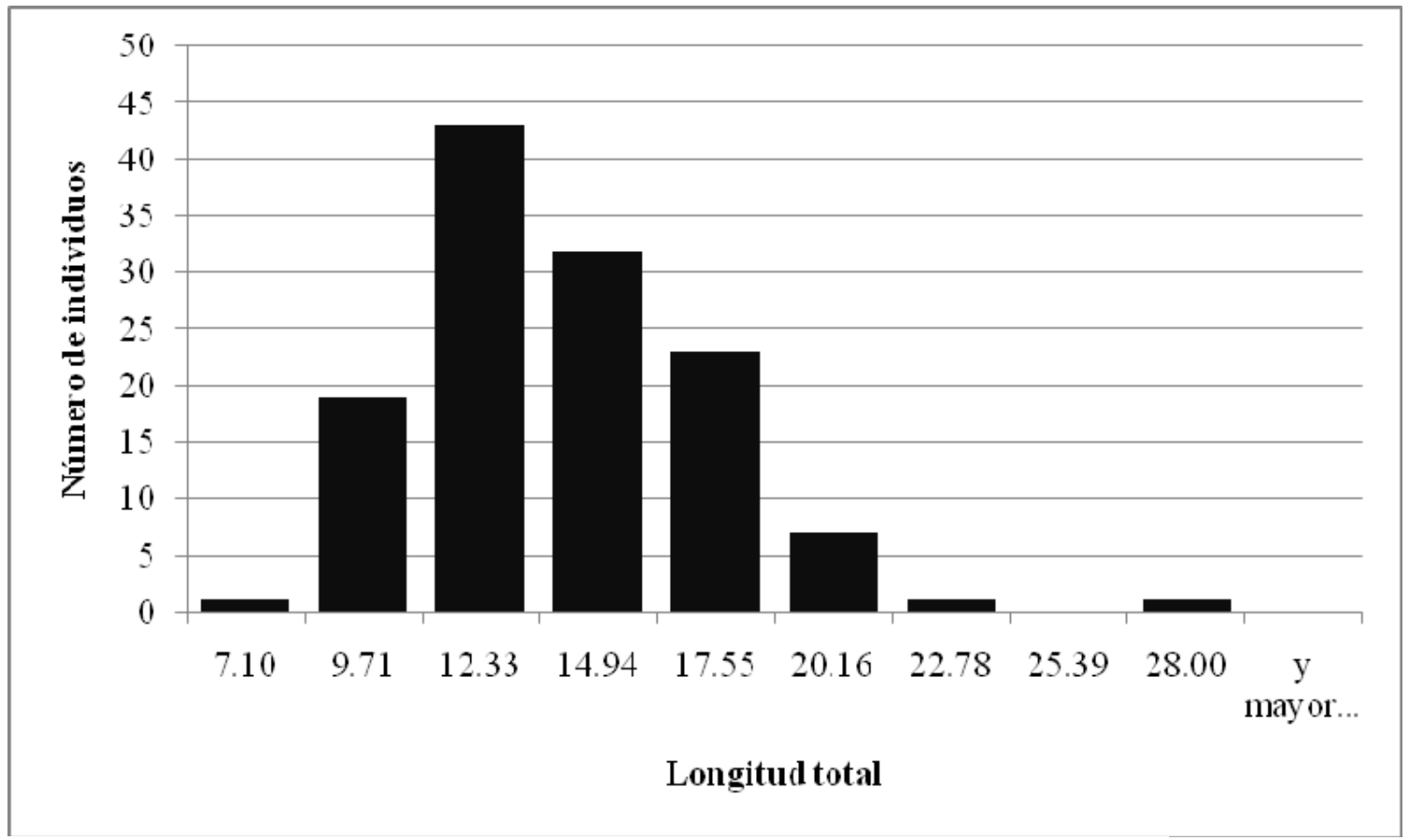

Figura 1. Frecuencia de las tallas de los individuos analizados. 


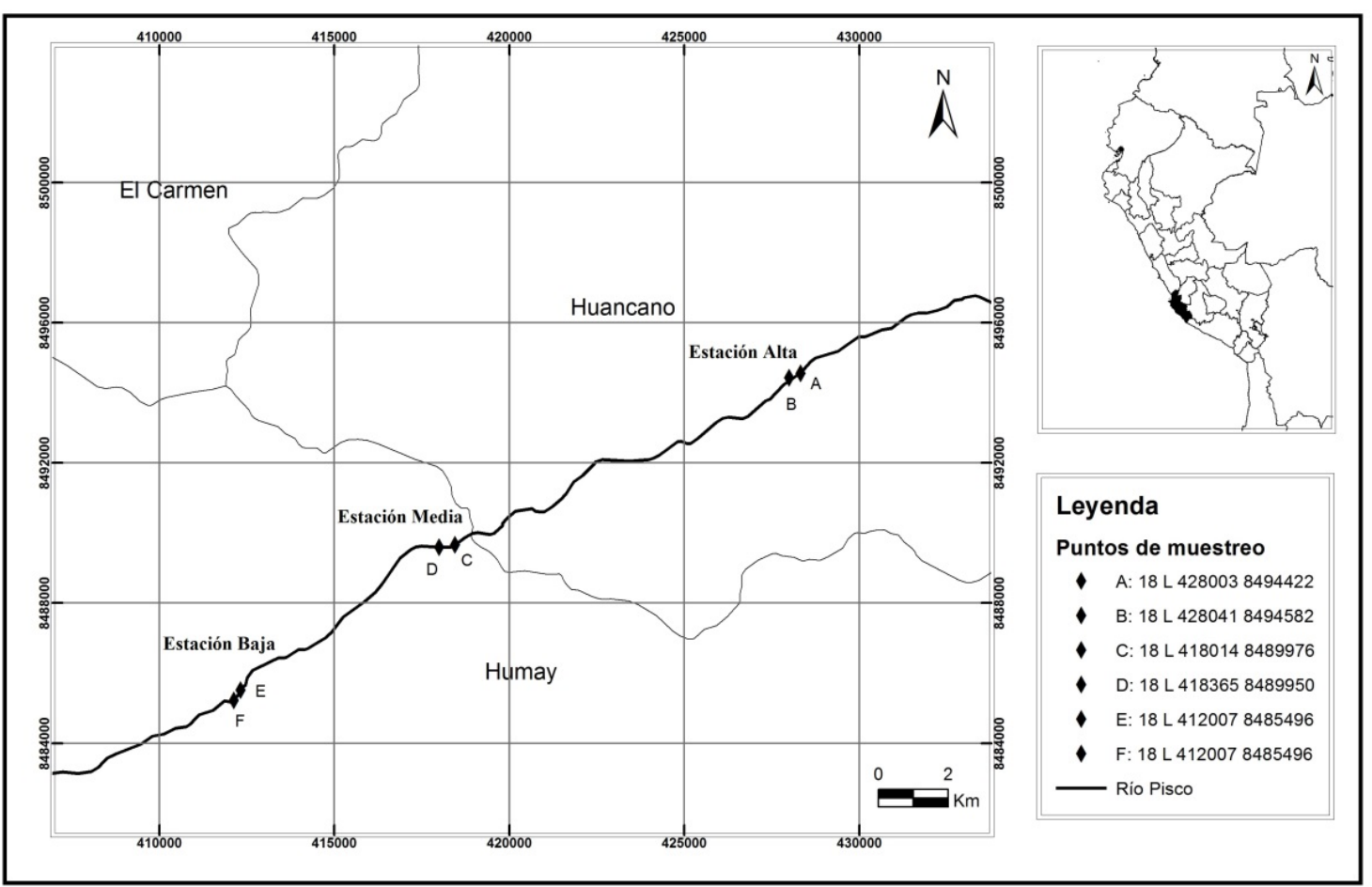

Figura 2. Mapa de los estaciones de colecta en el río Pisco (UTM WGS 84).

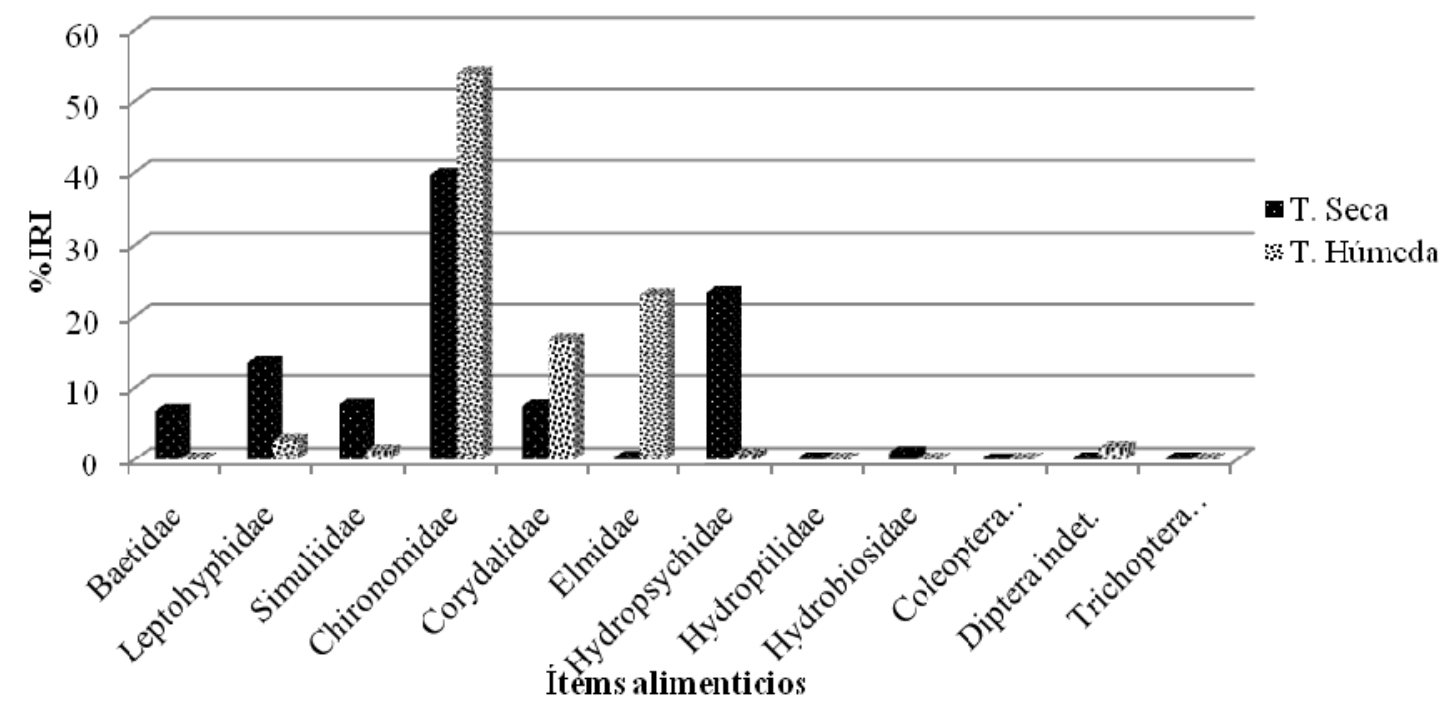

Figura 3. Porcentaje de Índice de Importancia Relativa (IRI) para T. seca (Agosto 2010) y T. húmeda (Mayo 2011). Río Pisco. Perú. 


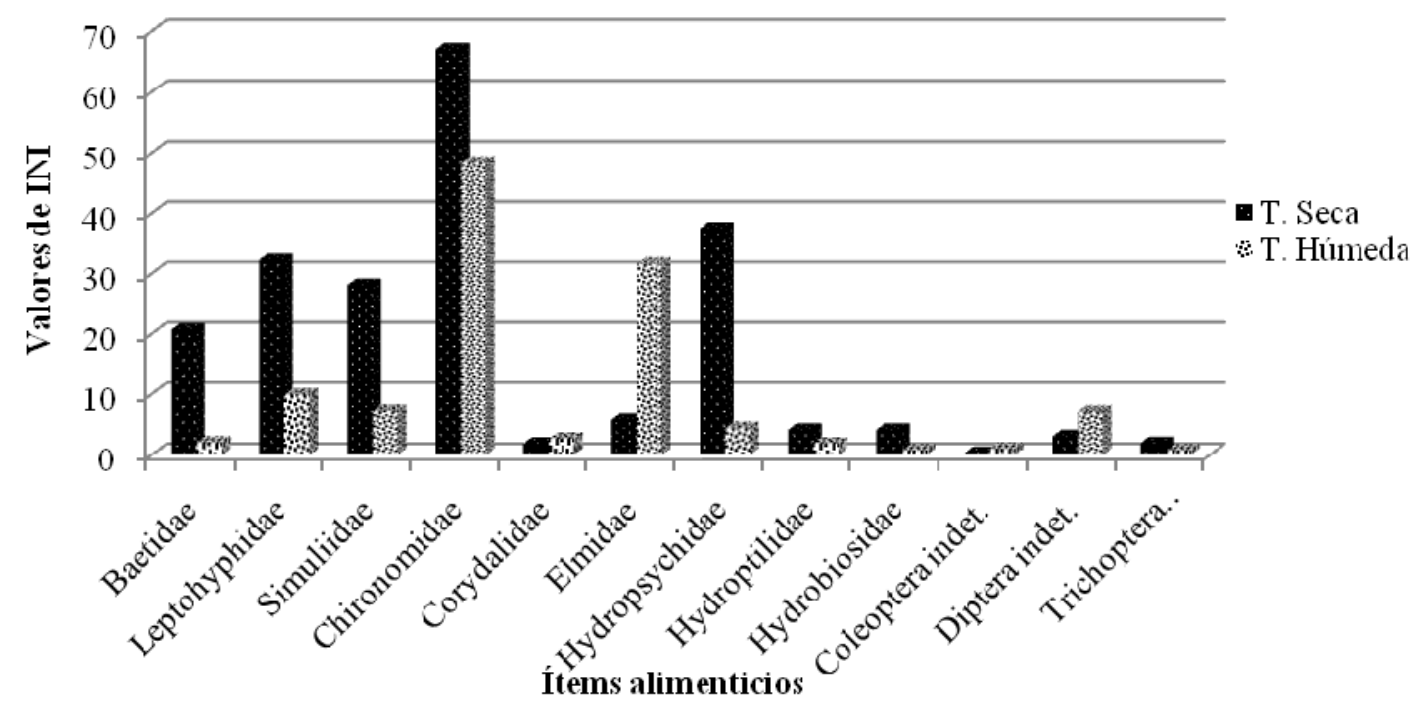

Figura 4. Índice de Importancia Numérica (INI) para T. seca (Agosto 2010) y T. húmeda (Mayo 2011). Río Pisco. Perú.

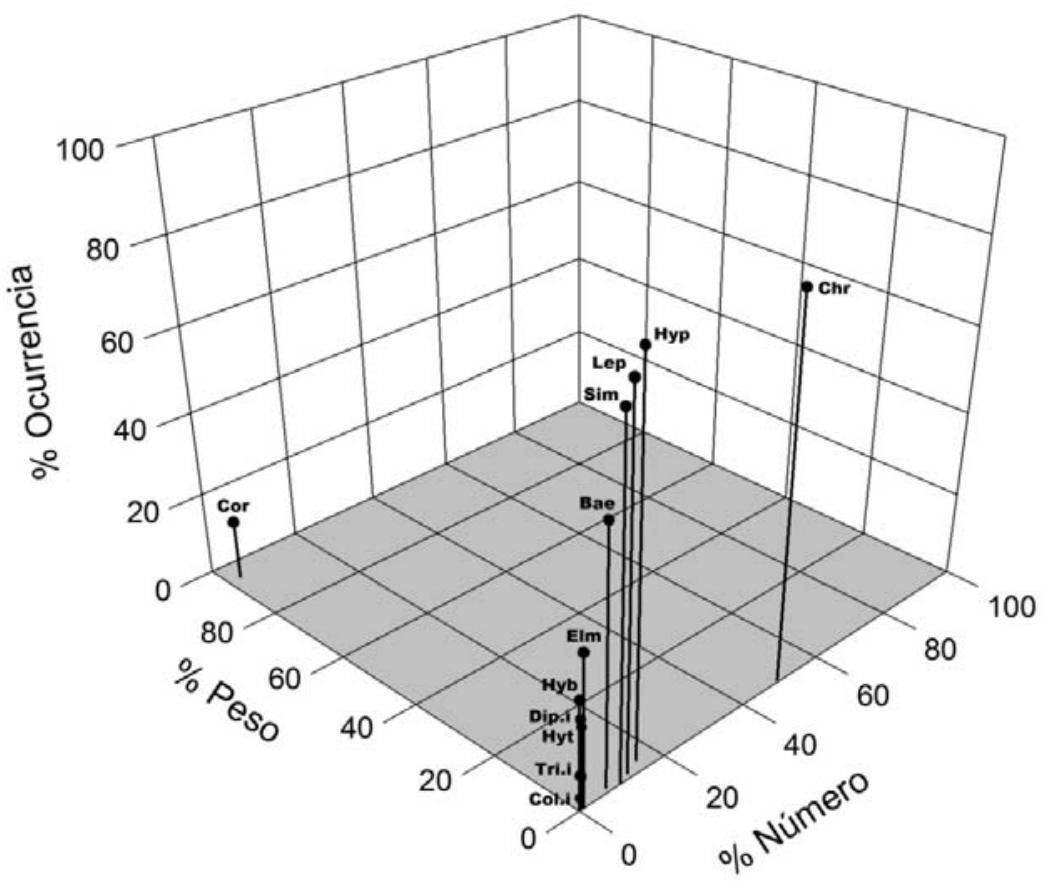

Figura 5. Gráfico de estrategia alimenticia en temporada seca (Agosto 2010). Río Pisco. Perú. 


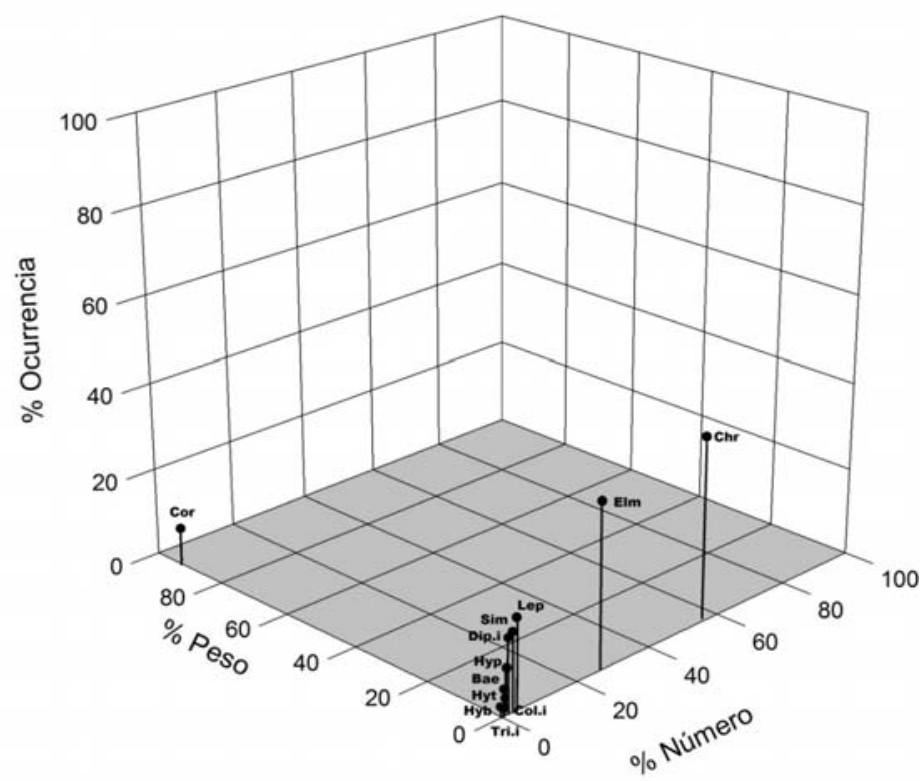

Figura 6. Gráfico de estrategia alimenticia en temporada húmeda (Mayo 2011). Río Pisco. Perú.

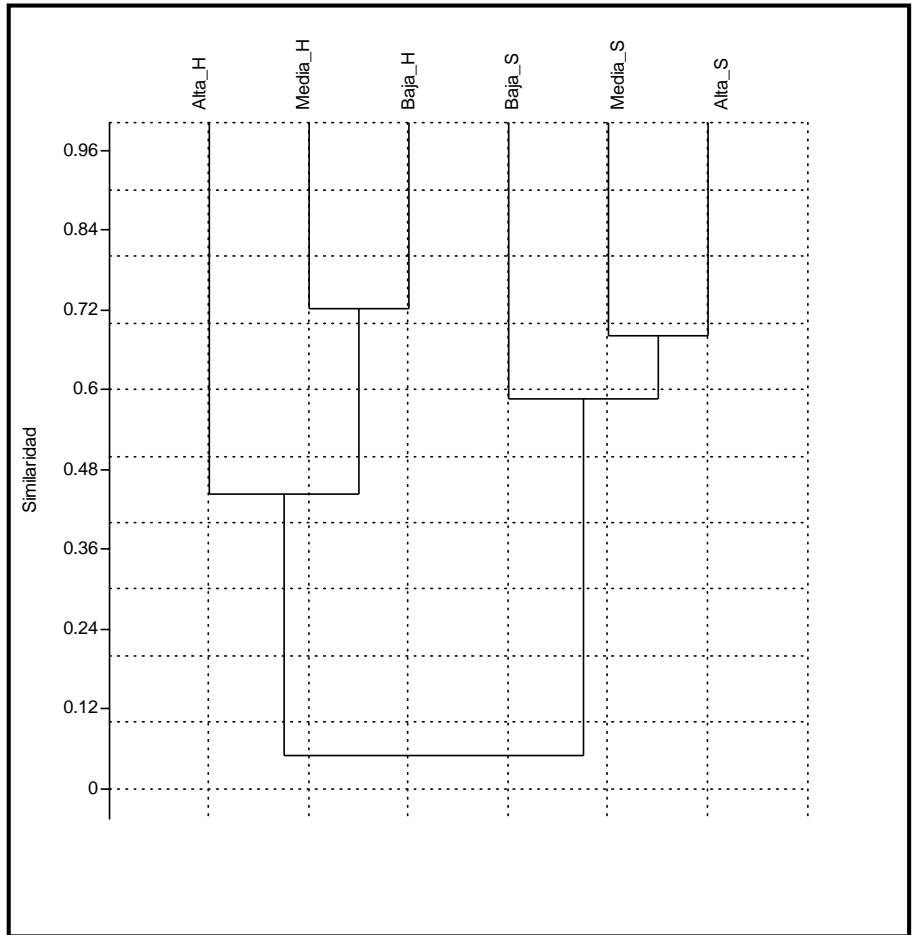

Figura 7. Gráfico de agrupamiento usando la medida de similitud de Bray-Curtis. 
${ }^{1}$ División de Limnología-Centro de Ornitología y Biodiversidad (CORBIDI).Calle Santa Rita N¹05 Of. 202 , Urb. Huertos de San Antonio, Santiago de Surco. Apartado postal 15-023. Lima- Perú. avera@corbidi.org. Laboratorio de Ecología de Procesos (LEP). Departamento Académico de Biología. Universidad Nacional Agraria La Molina. Av. La Molina S/N, La Molina. Apartado postal 15-024. Lima - Perú.

2 División de Limnología-Centro de Ornitología y Biodiversidad (CORBIDI). Calle Santa Rita N ${ }^{\circ} 105$ Of. 202 , Urb. Huertos de San Antonio, Santiago de Surco. Apartado postal 15-023. Lima - Perú.

Knight Piésold Consultores S.A. Área de Medio Ambiente. Calle Aricota $1065^{\text {to }}$ Piso, Santiago de Surco. Apartado postal 15-038, Lima - Perú. eoyague@knightpiesold.com

${ }^{3}$ Laboratorio de Ecología de Procesos (LEP). Departamento Académico de Biología. Universidad Nacional Agraria La Molina. Av. La Molina S/N. Apartado postal 15-024, Lima - Perú. lizcastaneda@lamolina.edu.pe·

${ }^{4}$ Laboratorio de Ecología de Procesos (LEP). Departamento Académico de Biología. Universidad Nacional Agraria La Molina. Av. La Molina S/N, La Molina. Apartado postal 15-024. Lima - Perú. zquinteros@lamolina.edu.pe 